\title{
Uso de torta de filtro enriquecida com fosfato natural e biofertilizantes em Latossolo Vermelho distrófico ${ }^{1}$
}

\author{
Leónides Castellanos González², Renato de Mello Prado ${ }^{3}$, \\ Alfredo Reyes Hernández ${ }^{4}$, Gustavo Caione ${ }^{3}$, Enrique Parets Selva ${ }^{2}$
}

\begin{abstract}
Use of filter cake enriched with rock phosphate and biofertilizers in a Haplustox soil

The use of biofertilizers associated with organic compound and phosphate rock can increase the availability of phosphorus in the soil, due to the increase of the microorganisms population. This study aimed at evaluating the effect of filter cake enriched with rock phosphate and biofertilizers preparations on the microorganisms population and phosphorus content of a Haplustox soil. The experiment was conducted in a greenhouse, in a completely randomized experimental design, in a $7 \times 3$ factorial scheme (seven treatments and three sampling times), with three replications. Treatments consisted of soil samples with decomposed filter cake and Araxá rock phosphate enriched with microorganisms from the biofertilizers Biopack, Embrafos and Azotofos; soil with filter cake and Araxá rock phosphate not enriched with microorganisms; soil with filter cake; soil with rock phosphate; and single soil (control). The soil phosphorus content and microorganisms population were evaluated at 30,60 and 90 days after the experiment installation. The use of filter cake enriched with rock phosphate and biofertilizers increased the soil bacteria population, with variations in time. The filter cake also increased the fungi population and phosphate solubilizing microorganisms. In the short term, the addition of filter cake enriched with rock phosphate contributed to the increase of the soil soluble phosphorus content.
\end{abstract}

KEY-WORDS: Organic residue; phosphate solubilizing microorganisms; phosphorus fertilization.

\section{INTRODUÇÃO}

Os solos tropicais possuem baixa disponibilidade de fósforo, sendo este fator reconhecido como um dos mais limitantes à produção agrícola (Prado 2008). Para elevar os teores de fósforo no solo, são necessárias aplicações de doses bem mais elevadas do

\section{RESUMO}

O uso de biofertilizantes associados a composto orgânico e rocha fosfatada pode aumentar a disponibilidade de fósforo no solo, pelo aumento da população de micro-organismos. Objetivou-se avaliar o efeito do uso da torta de filtro enriquecida com fosfato natural e preparados de biofertilizantes na população de micro-organismos e no teor de fósforo de um Latossolo Vermelho distrófico. O experimento foi realizado em casa-devegetação, utilizando-se delineamento experimental inteiramente casualizado, em esquema fatorial $7 \times 3$ (sete tratamentos e três épocas de avaliação), com três repetições. Os tratamentos consistiram de amostras de solo com adições de torta de filtro decomposta e rocha fosfatada de Araxá enriquecida com microorganismos provenientes dos biofertilizantes Biopack, Embrafos e Azotofos; solo com adições de torta de filtro e rocha fosfatada de Araxá, sem enriquecimento com micro-organismos; solo com torta de filtro; solo com rocha fosfatada; e somente solo (controle). Avaliou-se, aos 30, 60 e 90 dias após a instalação do experimento, o teor de fósforo disponível no solo e a população de micro-organismos. A presença de torta de filtro enriquecida com fosfato natural e com biofertilizantes promoveu aumento na população de bactérias do solo, havendo variações no tempo. A torta de filtro também proporcionou aumento na população de fungos e micro-organismos solubilizadores de fosfato. A curto prazo, a adição de torta de filtro enriquecida com fosfato natural contribuiu para o aumento do teor de fósforo solúvel no solo.

PALAVRAS-CHAVE: Resíduo orgânico; micro-organismos solubilizadores de fosfato; adubação fosfatada.

que a quantidade que as plantas necessitam, devido aos processos químicos de adsorção, induzindo à formação de fosfatos de cálcio em solos alcalinos e de fosfatos de ferro e de alumínio em solos ácidos.

A dinâmica do fósforo no solo está associada a fatores ambientais que controlam a atividade dos micro-organismos, os quais imobilizam ou liberam

1. Trabalho recebido em abr./2013 e aceito para publicação em abr./2014 (nº registro: PAT 23478).

2. Universidad de Cienfuegos, Centro de Estudios para la Transformación Agraria Sostenible (CETAS), Facultad de Ciencias

Agrarias, Cienfuegos, Cuba.E-mails: 1castellanos@ucf.edu.cu, eparets@ucf.edu.cu.

3. Universidade Estadual Paulista (Unesp), Departamento de Solos e Adubos, Jaboticabal, SP, Brasil. E-mails: rmprado@fcav.unesp.br, gustavocaione@agronomo.eng.br.

4. Universidad de Sancti Spiritus, Facultad Agropecuaria de Montaña del Escambray, Sancti Spiritus, Cuba. 
os íons ortofosfato, e às propriedades físico-químicas e mineralógicas do solo. Assim, os processos geoquímicos e biológicos transformam os fosfatos naturais em formas orgânicas e inorgânicas estáveis e transferem o fósforo entre os compartimentos do ambiente (Santos et al. 2008).

Estudos têm sido realizados visando a aumentar os teores de fósforo disponíveis para as plantas, por meio de processos microbiológicos, atributos que têm sido utilizados para avaliar o grau de sustentabilidade de um sistema agrícola (Bezerra et al. 2008). Neste sentido, nota-se tendência de maior atenção nestes estudos, nos países em desenvolvimento que não possuem reservas de rochas fosfatadas para a produção em larga escala do fertilizante comercial acidulado. Desta forma, torna-se importante o estudo de processos microbiológicos, como a solubilização de fosfato, que favoreçam a disponibilização de fósforo (Mendes \& Reis Júnior 2003).

Um fenômeno importante que ocorre com o fósforo inorgânico é a solubilização de fosfatos nativos ou precipitados e a mineralização de fósforo orgânico por micro-organismos. Os micro-organismos solubilizadores de fosfatos desempenham importante papel na disponibilização de formas inorgânicas de fosfatos (Ca-P, Al-P e Fe-P), resultando em aumento no teor de fósforo da solução (Chabot et al. 1993).

Diante dos elevados custos do processo de produção de fosfatos solúveis, tem-se incentivado o uso de fontes alternativas de fósforo (Prochnow et al. 2004). Os fosfatos naturais apresentam baixa disponibilidade de fósforo para as plantas a curto prazo, porém, com custo por unidade de fósforo menor que o dos fosfatos solúveis (Novais et al. 2007).

$\mathrm{O}$ uso de fertilizantes organominerais pode se tornar uma alternativa para reduzir os custos e fornecer fósforo em quantidades suficientes para as plantas, a partir da mistura do fosfato natural com uma fonte de carbono, como a torta de filtro (resíduo da indústria sucroalcooleira). Na literatura, os resultados sobre o uso de micro-organismos solubilizadores de fosfato são contraditórios, em experimentos de campo e casa-de-vegetação, pois a eficiência de solubilização depende da estirpe do micro-organismo, do fosfato, tipo de solo, cultivar, acidez e natureza das matérias orgânicas que servirão como fonte de carbono para os micro-organismos (Nahas et al. 1994a e 1994b, Nahas 2002). O estudo sobre a dinâmica populacional de micro-organismos no solo é outro fator importante, pois, após a incorporação de biofertilizante ao solo, ocorrem várias alterações na população microbiana, sendo importante o monitoramento em diferentes épocas de avaliação (Rodrigues et al. 2011).

Nesse sentido, tem-se utilizado a torta de filtro associada a fertilizante fosfatado, podendo-se reduzir a adubação fosfatada mineral solúvel, dependendo da dose de fósforo recomendada (Santos et al. 2010, Almeida Júnior et al. 2011). Porém, as informações sobre o efeito do uso do fosfato natural associado com a torta e micro-organismos, na disponibilidade de fósforo no solo e na população de micro-organismos, são incipientes, no Brasil. Existem dúvidas na literatura se os micro-organismos solubilizadores de fosfato seriam capazes de competir com outros estabelecidos na rizosfera (Richarson et al. 2009).

Objetivou-se, neste estudo, avaliar o efeito do uso da torta de filtro enriquecida com fosfato natural e preparados biofertilizantes na população de microorganismos e no teor de fósforo de um Latossolo Vermelho distrófico.

\section{MATERIAL E MÉTODOS}

A pesquisa foi realizada em condições de laboratório, na Universidade Estadual Paulista (Unesp), Campus de Jaboticabal (SP), de setembro a novembro de 2012. Utilizaram-se amostras de um Latossolo Vermelho distrófico (Embrapa 2006), no qual foi realizada análise química (Raij et al. 2001), para fins de fertilidade do solo, obtendo-se os seguinte resultados: $\mathrm{pH} \mathrm{em} \mathrm{CaCl}_{2}=6,4$; M.O. $=6 \mathrm{~g} \mathrm{dm}^{-3}$; P resina $=$ $5 \mathrm{mg} \mathrm{dm}^{-3} ; \mathrm{K}=0,4 \mathrm{mmol} \mathrm{dm}^{-3} ; \mathrm{Ca}=12 \mathrm{mmol} \mathrm{dm}^{-3}$; $\mathrm{Mg}=6 \mathrm{mmol}_{\mathrm{c}} \mathrm{dm}^{-3} ; \mathrm{H}+\mathrm{Al}=15 \mathrm{mmol}_{\mathrm{c}} \mathrm{dm}^{-3} ; \mathrm{SB}$ (soma de bases $)=18,4 \mathrm{mmol}_{\mathrm{c}} \mathrm{dm}^{-3}$; $\mathrm{CTC}^{\mathrm{c}}$ (capacidade de troca catiônica) $=33,4 \mathrm{mmol}_{\mathrm{c}} \mathrm{dm}^{-3}$; e V (saturação por bases) $=55 \%$.

O experimento foi constituído por sete tratamentos e três épocas de avaliação. Os tratamentos foram: amostras de solo com adições de torta de filtro decomposta e rocha fosfatada de Araxá enriquecida com micro-organismos provenientes dos biofertilizantes Biopack (1), Embrafos (2) e Azotofos (3); amostras de solo com adições de torta de filtro e rocha fosfatada de Araxá, sem enriquecimento com micro-organismos (4); solo com torta de filtro (5); solo com rocha fosfatada (6); e somente solo (controle) (7). As épocas de avaliação foram 30, 60 e 90 dias após a instalação do ensaio. $\mathrm{O}$ delineamento experimental utilizado foi o inteiramente ao acaso, 
com três repetições, em esquema fatorial $7 \times 3$. A unidade experimental foi composta de recipiente de polipropileno com capacidade para $0,20 \mathrm{dm}^{3}$.

Foram utilizados, como fonte de microorganismos, os biofertilizantes sólidos brasileiros Biopack Sc e Krion Agroscience, com carga microbiana de $10^{6} \mathrm{UFC} \mathrm{g}^{-1}$, e FF organic Plus e Embrafos, com carga microbiana de $10^{4} \mathrm{UFC} \mathrm{g}^{-1}$, bem como o Azotofos (Pseudomonas fluorescens e Azotobacterr chococcum), obtido no Laboratório Barajagua do Instituto de Pesquisas de Solos e Adubos de Cuba, com carga microbiana de $10^{6} \mathrm{UFC}^{-1}$. A escolha destes biofertilizantes se deu pela disponibilidade de tais produtos e com o objetivo de testar a eficiência de dois biofertilizantes comercializados no Brasil e outro comercializado em Cuba, onde esta técnica é mais utilizada e conhecida.

Efetuou-se a contagem de micro-organismos presentes na torta de filtro a partir do procedimento de diluição em série (Wollum 1982). Para a contagem de bactérias totais, utilizou-se o meio de cultivo proposto por Bunt \& Rovira (1955), com pH 7,4. Para a contagem de fungos totais, utilizou-se o meio de cultivo de Martin (1950), com pH 5,6, e, para a quantificação dos micro-organismos solubilizadores de fosfato, o meio de cultivo utilizado foi o de Pikoskaya (Martínez Vieira et al. 2006). Os resultados para bactérias totais, micro-organismos solubilizadores de fosfato e fungos totais foram, respectivamente, $2,97 \mathrm{x}$ $10^{5} \mathrm{UFC} \mathrm{g}^{-1}, 2,5 \times 10^{2} \mathrm{UFC} \mathrm{g}^{-1}$ e $2,5 \times 10^{3} \mathrm{UFC} \mathrm{g}^{-1}$.

Realizou-se a análise química da torta de filtro conforme metodologia proposta por Bataglia et al. (1983), obtendo-se os seguintes resultados: $\mathrm{N}=1,40 \% ; \mathrm{P}=1,17 \% ; \mathrm{K}=0,22 \% ; \mathrm{Ca}=2,74 \%$; $\mathrm{Mg}=1,08 \%$; e S $=0,24 \%$. Avaliou-se, ainda, o pH $\left(\mathrm{CaCl}_{2}\right)(8,2)$ e o teor de M.O. total $(29,6 \%)$.

As doses de biofertilizantes foram equivalentes a $692 \mathrm{~kg} \mathrm{ha}^{-1}$ para o Biopack, $500 \mathrm{~kg} \mathrm{ha}^{-1}$ para o Embrafos e $70 \mathrm{~kg} \mathrm{ha}^{-1}$ para o Azotofos, de acordo com indicações dos fabricantes. Antes da aplicação, todos os biofertilizantes foram ativados durante 12 horas, com circulação de ar, em presença de $0,4 \mathrm{~kg}$ de torta e $17,5 \mathrm{~g}$ de rocha fosfatada por litro de água. O processo de ativação dos micro-organismos é sugerido por Martínez Vieira et al. (2006) e utilizado por algumas usinas sucroalcooleiras que aplicam a torta de filtro enriquecida no sulco de plantio da cana-de-açúcar. A dose de torta utilizada (enriquecida ou não com micro-organismos) correspondeu a $25 \mathrm{t} \mathrm{ha}^{-1}(12,5 \mathrm{~g}$ de torta $\mathrm{dm}^{-3}$ de solo). Depois de ter sido aplicada a torta enriquecida ou não, o solo foi homogeneizado. A dose de fósforo empregada foi de $60 \mathrm{mg} \mathrm{dm}^{-3} \mathrm{de} \mathrm{P}$ solúvel em ácido cítrico, na forma de fosfato natural de Araxá ( $4 \%$ de $\mathrm{P}_{2} \mathrm{O}_{5}$ solúvel em ácido cítrico).

Em todos os tratamentos, foram aplicados, de maneira uniforme, nitrogênio $\left(200 \mathrm{mg} \mathrm{dm}^{-3}\right)$, na forma de ureia $(45 \%$ de $\mathrm{N})$, e potássio $\left(150 \mathrm{mg} \mathrm{dm}^{-3}\right)$, na forma de cloreto de potássio $\left(60 \%\right.$ de $\left.\mathrm{K}_{2} \mathrm{O}\right)$. A irrigação foi realizada diariamente, mantendo-se o solo a $60 \%$ da capacidade de retenção de água.

Foi realizada uma amostragem de solo aos 30, 60 e 90 dias após a instalação do experimento, para a posterior determinação do teor de fósforo disponível no solo (Raij et al. 2001). Nestes mesmos períodos, avaliaram-se as populações de micro-organismos (bactérias totais, micro-organismos solubilizadores de fosfato e fungos totais), utilizando-se a mesma metodologia aplicada na análise da torta.

As placas foram incubadas à temperatura de $30^{\circ} \mathrm{C}$, por 48 horas (bactérias), 72 horas (fungos) e 96 horas (micro-organismos solubilizadores de fosfato), e, em seguida, foram avaliadas em um contador de colônias com $6 \mathrm{x}$ de aumento, considerando-se apenas as contagens que variaram de 30 a 300 colônias.

Os dados foram submetidos à análise de variância e as médias comparadas pelo teste Tukey $(\mathrm{p}<0,05)$, utilizando-se o programa estatístico Assistat, versão 7.6 beta (Silva \& Azevedo 2006). Para as populações de micro-organismos, a análise de variância foi realizada com base nos dados transformados em logaritmo.

\section{RESULTADOS E DISCUSSÃO}

Avaliando-se o número de bactérias e fungos totais, micro-organismos solubilizadores de fosfato e teor de fósforo disponível no solo, verificou-se efeito significativo da interação entre tratamentos e épocas de avaliação sobre todas as variáveis (Tabela 1).

As populações de bactérias totais apresentaram-se mais elevadas aos 30 dias após a instalação do ensaio, no tratamento com a presença da torta de filtro, fosfato natural e Biopack, e no tratamento com torta de filtro, fosfato natural e Azotofos, enquanto, no tratamento com torta de filtro, fosfato natural e Embrafos, houve aumento aos 60 e aos 90 dias (Tabela 2).

Isso ocorreu devido ao fato de a carga mirobiana do Embrafos $\left(10^{4} \mathrm{UFC} \mathrm{g}^{-1}\right)$ ser menor que a do Biopack $\left(10^{6} \mathrm{UFC} \mathrm{g}^{-1}\right)$ e do Azotofos $\left(10^{6} \mathrm{UFC} \mathrm{g}^{-1}\right)$. 
Tabela 1. Unidades formadoras de colônias (UFC) de bactérias e fungos totais, micro-organismos solubilizadores de fosfato e teor de fósforo disponível no solo, em função da aplicação de torta de filtro enriquecida com fosfato natural de Araxá e biofertilizantes, em diferentes épocas de avaliação (Jaboticabal, SP, 2012).

\begin{tabular}{|c|c|c|c|c|}
\hline \multirow{3}{*}{ Épocas (E) } & Bactérias totais & Fungos totais & $\begin{array}{c}\text { Micro-organismos } \\
\text { solubilizadores }\end{array}$ & \multirow{2}{*}{$\frac{\text { P no solo }}{\left(\mathrm{mg} \mathrm{dm}^{-3}\right)}$} \\
\hline & \multicolumn{3}{|c|}{ - UFC g g ${ }^{-1}$ solo seco } & \\
\hline & $\mathrm{x} 10^{7}$ & $\mathrm{x} 10^{4}$ & x $10^{4}$ & \\
\hline 30 dias & 5,20 & 3,28 & 3,73 & 28,70 \\
\hline 60 dias & 5,09 & 2,92 & 3,96 & 32,00 \\
\hline 90 dias & 5,00 & 2,96 & 3,57 & 30,00 \\
\hline \multicolumn{5}{|l|}{ Tratamentos $(\mathrm{T})$} \\
\hline Torta + Araxá + Biopack & 5,99 & 3,62 & 4,44 & 39,50 \\
\hline Torta + Araxá + Embrafos & 5,60 & 3,53 & 3,75 & 41,10 \\
\hline Torta + Araxá + Azotofos & 5,97 & 3,46 & 4,62 & 48,50 \\
\hline Torta + Araxá & 5,33 & 3,19 & 3,75 & 46,20 \\
\hline Torta & 5,03 & 3,16 & 3,84 & 22,20 \\
\hline Araxá & 4,08 & 2,28 & 2,98 & 8,50 \\
\hline Controle & 4,03 & 2,28 & 2,98 & 5,40 \\
\hline & \multicolumn{4}{|c|}{ Teste $F$} \\
\hline Épocas (E) & $22,51 * *$ & $8,79 * *$ & $18,80 * *$ & $2,83^{\mathrm{ns}}$ \\
\hline Tratamentos $(\mathrm{T})$ & $637,05 * *$ & $35,95 * *$ & $87,30 * *$ & $145,11 * *$ \\
\hline $\mathrm{E} \times \mathrm{T}$ & $14,55 * *$ & $3,81 * *$ & $21,72 * *$ & $3,06 * *$ \\
\hline CV (\%) & 1,90 & 2,80 & 5,40 & 14,90 \\
\hline
\end{tabular}

${ }_{\text {ns }}$ não significativo; $* *$ significativo a $1 \%$.

Tabela 2. População de bactérias totais, fungos totais e micro-organismos solubilizadores de fosfato no solo, em diferentes épocas de avaliação, em função da aplicação de torta de filtro enriquecida com fosfato natural de Araxá e biofertilizantes (Jaboticabal, SP, 2012).

\begin{tabular}{|c|c|c|c|}
\hline \multirow{2}{*}{ Tratamentos } & \multicolumn{3}{|c|}{ Épocas } \\
\hline & 30 dias & 60 dias & 90 dias \\
\hline & \multicolumn{3}{|c|}{ Bactérias totais (UFC x $10^{7} \mathrm{~g}^{-1}$ solo seco) } \\
\hline Torta + Araxá + Biopack & $6,18 \mathrm{aA}$ & $5,93 \mathrm{bA}$ & $5,85 \mathrm{bA}$ \\
\hline Torta + Araxá + Embrafos & $5,38 \mathrm{bB}$ & $5,62 \mathrm{aB}$ & $5,80 \mathrm{aA}$ \\
\hline Torta + Araxá + Azotofos & $6,19 \mathrm{aA}$ & $5,86 \mathrm{bA}$ & $5,82 \mathrm{bA}$ \\
\hline Torta + Araxá & $5,37 \mathrm{aB}$ & $5,05 \mathrm{aC}$ & $4,82 \mathrm{bB}$ \\
\hline Torta & $5,22 \mathrm{aB}$ & $5,04 \mathrm{bC}$ & $4,53 \mathrm{bC}$ \\
\hline Araxá & $4,04 \mathrm{aC}$ & $4,07 \mathrm{aD}$ & $4,13 \mathrm{aD}$ \\
\hline \multirow[t]{2}{*}{ Controle } & $4,06 \mathrm{aC}$ & $3,98 \mathrm{aD}$ & $4,07 \mathrm{aD}$ \\
\hline & \multicolumn{3}{|c|}{ Fungos totais (UFC $\times 10^{4} \mathrm{~g}^{-1}$ solo seco) } \\
\hline Torta + Araxá + Biopack & $4,24 \mathrm{aA}$ & $3,12 \mathrm{bA}$ & $3,48 \mathrm{bA}$ \\
\hline Torta + Araxá + Embrafos & $3,97 \mathrm{aAB}$ & $3,22 \mathrm{bA}$ & $3,39 \mathrm{abAB}$ \\
\hline Torta + Araxá + Azotofos & $3,85 \mathrm{aAB}$ & $3,22 \mathrm{bA}$ & $3,31 \mathrm{abAB}$ \\
\hline Torta + Araxá & $3,68 \mathrm{aAB}$ & $3,12 \mathrm{abA}$ & $3,28 \mathrm{aAB}$ \\
\hline Torta & $3,25 \mathrm{aB}$ & $3,05 \mathrm{aA}$ & $2,70 \mathrm{bBC}$ \\
\hline Araxá & $1,93 \mathrm{aC}$ & $2,49 \mathrm{aAB}$ & $2,34 \mathrm{aC}$ \\
\hline \multirow[t]{2}{*}{ Controle } & $2,02 \mathrm{aC}$ & $2,25 \mathrm{aB}$ & $2,26 \mathrm{aC}$ \\
\hline & \multicolumn{3}{|c|}{ Micro-organismos solubilizadores (UFC $\times 10^{4} \mathrm{~g}^{-1}$ solo seco) } \\
\hline Torta + Araxá + Biopack & $5,19 \mathrm{aA}$ & $4,35 \mathrm{bA}$ & $3,80 \mathrm{cA}$ \\
\hline Torta + Araxá + Embrafos & $3,14 \mathrm{cBCD}$ & $4,32 \mathrm{aA}$ & $3,79 \mathrm{bA}$ \\
\hline Torta + Araxá + Azotofos & $5,65 \mathrm{aA}$ & $4,37 \mathrm{bA}$ & $3,86 \mathrm{cA}$ \\
\hline Torta + Araxá & $3,51 \mathrm{bB}$ & $4,01 \mathrm{aA}$ & $3,72 \mathrm{bA}$ \\
\hline Torta & $3,24 \mathrm{bBC}$ & $4,30 \mathrm{aA}$ & $3,98 \mathrm{aA}$ \\
\hline Araxá & $2,73 \mathrm{aCD}$ & $3,15 \mathrm{aB}$ & $2,81 \mathrm{aB}$ \\
\hline Controle & $2,65 \mathrm{bD}$ & $3,26 \mathrm{aB}$ & $3,04 \mathrm{abB}$ \\
\hline
\end{tabular}

Médias seguidas pela mesma letra, maiúscula na coluna e minúscula na linha, não diferem entre si, pelo teste Tukey, a 5\%. UFC g-1: unidades formadoras de colônias por grama de solo seco. 
Portanto, no tratamento com Biopack e Azotofos, houve elevado aporte inicial de micro-organismos e, posteriormente, houve competição entre eles e outros nativos, ocasionando morte, conforme ressaltado por Nahas et al. (1994a, 1994b). O tratamento que recebeu torta + fosfato de Araxá apresentou valores semelhantes, aos 30 e 60 dias. Os tratamentos com ausência de torta não apresentaram diferenças, em relação ao tempo de avaliação.

Para os efeitos dos tratamentos, os biofertilizantes Biopack e Azotofos apresentaram valores de bactérias totais, aos 30 e 60 dias, superiores aos dos demais tratamentos (Tabela 2), devido à maior carga microbiana destes biofertilizantes. Aos 90 dias, houve maior população de bactérias totais em todos os tratamentos com a presença de biofertilizantes, seguidos do tratamento com a torta sem enriquecimento com biofertilizantes. Os tratamentos com ausência de torta apresentaram valores de bactérias totais inferiores aos dos tratamentos com a presença da mesma, em todas as épocas de avaliação, o que demonstra a importância da torta de filtro para a população microbiana do solo (Cardoso et al. 1995).

Para a população de fungos totais, houve diminuição no tempo, em especial para os tratamentos que usaram torta de filtro e biofertilizantes aos 60 dias, o que demonstra a diminuição do substrato neste período em que pode ter ocorrido crescimento acelerado dos micro-organismos nos primeiros 30 dias de condução do ensaio, ocasionando redução na população destes micro-organismos aos 60 dias, devido à competição (Tabela 2). Para os tratamentos que não receberam torta de filtro, não houve grandes variações em função da época de avaliação.

Para o efeito dos tratamentos dentro de cada época de avaliação, de modo geral, houve maior número de fungos totais nos tratamentos com a utilização da torta de filtro enriquecida com biofertilizantes e com fosfato de rocha, seguidos do tratamento apenas com torta de filtro, em todas as épocas avaliadas (Tabela 2). Os tratamentos sem o uso da torta de filtro apresentaram menores números de fungos em todas as épocas.

É importante ressaltar que os biofertilizantes não promoveram aumento na população de fungos, mas sim a torta e o fosfato de rocha, já que os tratamentos enriquecidos com diferentes biofertilizantes não diferiram do tratamento com torta e rocha fosfatada, em todas as épocas avaliadas. Nahas et al. (1994c) também verificaram aumento no número de fungos, com a adição de matéria orgânica (palha de milho moída), em relação ao tratamento com aplicação isolada de rocha fosfatada, em Latossolo Vermelho escuro.

Houve diminuição na população de microorganismos solubilizadores de fosfato, com o tempo de avaliação (Tabela 2), nos tratamentos com torta enriquecida com fosfato natural e Biopack e com Azotofos. Este efeito se deve, provavelmente, ao maior aporte inicial destes micro-organismos, apresentando rápido crescimento inicial e, posteriormente, ocorrendo competição, diminuindo a população. Nos tratamentos com Embrafos, torta enriquecida com fosfato natural e apenas torta, o crescimento da população de micro-organismos solubilizadores de fosfato mostrou-se mais lento, havendo maiores valores aos 60 dias. Para o tratamento sem a presença de torta de filtro e com fosfato de Araxá, as populações mantiveram-se semelhantes.

Para o efeito dos tratamentos dentro de cada época de avaliação (Tabela 2), de modo geral, houve maior número de micro-organismos solubilizadores de fosfato nos tratamentos com a utilização da torta de filtro, independentemente da presença de biofertilizante, exceto aos 30 dias, em que os tratamentos com torta enriquecida com Azotofos e Biopack apresentaram maior número, o qual se explica pela maior adição de micro-organismos solubilizadores destes biofertilizantes. Porém, com o passar do tempo, este número tende a se igualar em todos os tratamentos com torta, o que pode ser reflexo da maior competitividade entre os micro-organismos deste material orgânico e os nativos do solo (Nahas et al. 1994a, 1994b).

Nos tratamentos com ausência de torta (solo com fosfato natural e apenas solo), as populações de micro-organismos solubilizadores de fosfato foram menores em todas as épocas de avaliação. O maior número de micro-organismos solubilizadores de fosfato, nos tratamentos com torta, pode ser atribuído aos vários benefícios da torta de filtro à qualidade do solo, devido às propriedades da matéria orgânica. $\mathrm{O}$ aumento no teor de matéria orgânica foi verificado aos 90 dias, por meio da análise química do solo, em que o resultado foi de $7,3 \mathrm{mg} \mathrm{dm}^{-3}$, nos tratamentos com ausência de torta de filtro, e de $8,5 \mathrm{mg} \mathrm{dm}^{-3}$, nos tratamentos com torta. Nahas et al. (1994c), estudando o efeito da inoculação de fungo solubilizador de fosfato aplicado em solo cultivado com milho, também verificaram que, nos tratamentos em que 
Tabela 3. Teor de fósforo disponível (resina) no solo, em diferentes épocas de avaliação, em função da aplicação de torta de filtro enriquecida com fosfato natural de Araxá e biofertilizantes (Jaboticabal, SP, 2012).

\begin{tabular}{|c|c|c|c|}
\hline \multirow{3}{*}{ Tratamentos } & \multicolumn{3}{|c|}{ Épocas } \\
\hline & 30 dias & 60 dias & 90 dias \\
\hline & \multicolumn{3}{|c|}{$\longrightarrow \mathrm{P}\left(\mathrm{mg} \mathrm{dm}^{-3}\right) \longrightarrow$} \\
\hline Torta + Araxá + Biopack & $43,3 \mathrm{aA}$ & $37,3 \mathrm{aC}$ & $38,0 \mathrm{aAB}$ \\
\hline Torta + Araxá + Embrafos & $41,0 \mathrm{aA}$ & $41,3 \mathrm{aBC}$ & $41,0 \mathrm{aAB}$ \\
\hline Torta + Araxá + Azotofos & $38,7 \mathrm{bA}$ & $56,4 \mathrm{aA}$ & $50,3 \mathrm{aA}$ \\
\hline Torta + Araxá & $39,7 \mathrm{bA}$ & $53,4 \mathrm{aA}$ & $45,7 \mathrm{abAB}$ \\
\hline Torta & $23,7 \mathrm{aB}$ & $20,0 \mathrm{aD}$ & $15,0 \mathrm{aC}$ \\
\hline Araxá & $10,3 \mathrm{aBC}$ & $11,7 \mathrm{aD}$ & $10,0 \mathrm{aD}$ \\
\hline Controle & $5,7 \mathrm{aC}$ & $5,3 \mathrm{aD}$ & $5,3 \mathrm{aD}$ \\
\hline
\end{tabular}

Médias seguidas pela mesma letra maiúscula, na coluna, e minúscula, na linha, não diferem entre si, pelo teste Tukey, a 5\%.

foi adicionada matéria orgânica, houve aumento no número de bactérias e fungos, em relação ao tratamento apenas com solo.

Na literatura, são limitadas as informações sobre a dinâmica dos micro-organismos solubilizadores de fosfato que são aportados pelos biofertilizantes e pela torta de filtro e sua capacidade competitiva no solo, necessitando-se, portanto, de mais pesquisas a respeito, especialmente a médio e longo prazo.

Os níveis de $\mathrm{P}$ mantiveram-se semelhantes no tempo, exceto no tratamento com torta enriquecida com fosfato de rocha e Azotofos e apenas torta e fosfato de rocha, que apresentaram aumento (Tabela 3 ). A dinâmica do P no solo é bastante complexa, sofrendo influência de vários fatores, como o $\mathrm{pH}$, presença de outros elementos químicos, teor e tipo de argila, entre outros. Assim, variações significativas no teor de P da solução do solo, em função do tempo, normalmente, demandam maior tempo.

Para o efeito dos tratamentos dentro de cada época de avaliação (Tabela 3), aos 30 dias, o teor de P no solo foi maior para os tratamentos com o uso da torta enriquecida com fosfato natural + micro-organismos e para o tratamento com torta + fosfato. Aos 60 e 90 dias, os maiores teores de $\mathrm{P}$ corresponderam aos tratamentos com torta enriquecida com fosfato natural e Azotofos e apenas torta e fosfato natural, embora, aos 90 dias, esta variável, nos tratamentos com torta enriquecida com Biopack e Embrafos, tenha apresentado resultado semelhante. O teor de P no solo dos tratamentos sem torta de filtro foi inferior ao dos tratamentos com torta, nas três épocas de avaliação (30, 60, e 90 dias). As variações nos teores de P no solo, nas diferentes épocas avaliadas nos tratamentos com adição de biofertilizantes, podem ser atribuídas à mobilização de P pela biomassa microbiana do solo.
É importante destacar que, aos 90 dias, o teor de $\mathrm{P}$ no solo elevou-se de $5,3 \mathrm{mg} \mathrm{dm}^{-3}$ (tratamento-controle) para 50,3 $\mathrm{mg} \mathrm{dm}^{-3}$ de $\mathrm{P}$ (torta enriquecida com fosfato de rocha e Azotofos), correspondendo a um aumento de 9,5 vezes, fato atribuído à utilização do composto orgânico (torta de filtro) na adubação, o que contribuiu para diminuir os processos de adsorção de $\mathrm{P}$, conforme comprovado por outros autores (Nziguheba et al. 1998, Bittencourt et al. 2006, Santos et al. 2010, Almeida Júnior et al. 2011). Acrescenta-se, a isto, o teor de $\mathrm{P}$ contido neste composto orgânico $(1,17 \%)$, que, na dose aplicada $(12,5 \mathrm{~g}$ de torta $\mathrm{dm}^{-3}$ de solo), correspondeu a $146 \mathrm{mg} \mathrm{dm}^{-3} \mathrm{de}$ P.

\section{CONCLUSÕES}

1. A presença de torta de filtro enriquecida com fosfato natural e com biofertilizantes promoveu aumento na população de bactérias do solo, havendo variações no tempo.

2. A torta de filtro proporcionou aumento na população de fungos e micro-organismos solubilizadores de fosfato.

3. A curto prazo, a adição de torta de filtro enriquecida com fosfato natural contribuiu para o aumento do teor de fósforo solúvel no solo, com pouca variação no tempo, não havendo aumento nos tratamentos com o uso de biofertilizantes.

\section{AGRADECIMENTOS}

À Coordenação de Aperfeiçoamento de Pessoal de Nível Superior (Capes), pela concessão de auxílio referente ao Programa de Cooperação Internacional Capes/MES Brasil-Cuba. 


\section{REFERÊNCIAS}

ALMEIDA JÚNIOR, A. B. et al. Fertilidade do solo e absorção de nutrientes em cana-de-açúcar fertilizada com torta de filtro. Revista Brasileira de Engenharia Agrícola e Ambiental, Campina Grande, v. 15, n. 10, p. 1004-1013, 2011.

BATAGLIA, O. C. et al. Métodos de análise química de plantas. Campinas: Instituto Agronômico, 1983. (Boletim técnico, 78).

BEZERRA, R. G. D. et al. Atividade microbiana em solo cultivado com cana-de-açúcar submetido a doses de fósforo. Revista Verde, Mossoró, v. 3, n. 4, p. 64-69, 2008.

BITTENCOURT, V. C. et al. Torta de filtro enriquecida. Revista Idea News, Ribeirão Preto, n. 63, p. 2-6, 2006.

BUNT, J. S.; ROVIRA, A. D. Microbiological studies of some subantartic soils. Journal of Soil Science, Oxford, v. 6, n. 1, p. 119-128, 1955.

CARDOSO, E. J. B. N.; JAHNEL, M. C.; MELLONI, R. Avaliação da maturação do composto de lixo urbano. In: CONGRESSO BRASILEIRO DE CIÊNCIA DO SOLO, 25., 1995, Viçosa. Anais... Viçosa: Sociedade Brasileira de Ciência do Solo, 1995. p. 2297.

CHABOT, R.; ANTOUN, H.; CESCAS, M. P. Stimulation de la croissance du mais et de la laitue romaine par dês microorganismes dissolvant le phosphore inorganique. Canadian Journal of Microbiology, Ottawa, v. 39, n. 10, p. 941-947, 1993.

EMPRESA BRASILEIRA DE PESQUISA AGROPECUÁRIA (Embrapa). Centro Nacional de Pesquisa de Solos. Sistema brasileiro de classificação de solos. Rio de Janeiro: Embrapa, 2006.

MARTIN, J. P. Use of acid, rose bengal, and streptomycin in the plate method for estimating soil fungi. Soil Science, Baltimore, v. 69, n. 2, p. 215-232, 1950.

MARTÍNEZ VIERA, R. et al. Procedimientos para el estudio y fabricación de biofertilizantes bacterianos. Maracay: Instituto Nacional de Investigaciones Agrícolas, 2006.

MENDES, I. C.; REIS JÚNIOR, F. B. Micro-organismos e disponibilidade de fósforo $(P)$ nos solos: uma análise crítica. Planaltina, DF: Embrapa, 2003.

NAHAS, E. Micro-organismos do solo produtores de fosfatases em diferentes sistemas agrícolas. Bragantia, Campinas, v. 61, n. 3, p. 267-275, 2002.

NAHAS, E.; CENTURION, J. F.; ASSIS, L. C. Microorganismos solubilizadores de fosfato e produtores de fosfatases de vários solos. Revista Brasileira de Ciência do Solo, Campinas, v. 18, n. 1, p. 43-48, 1994a.

NAHAS, E.; CENTURION, J. F.; ASSIS, L. C. Efeito das características químicas dos solos sobre os micro- organismos solubilizadores de fosfato e produtores de fosfatases. Revista Brasileira de Ciência do Solo, Campinas, v. 18, n. 1, p. 49-53, 1994b.

NAHAS, E.; FORNASIEREI, D. J.; ASSIS, L. C. Resposta à inoculação de fungo solubilizador de fósforo em milho. Scientia Agricola, Piracicaba, v. 51, n. 3, p. 463-469, 1994c.

NOVAIS, R. F.; SMYTH, T. J.; NUNES, F. N. Fósforo. In: NOVAIS, R. F. et al. (Eds.). Fertilidade do solo. Viçosa: SBCS, 2007.

NZIGUHEBA, G. et al. Soil phosphorus fractions and adsorption as affected by organic and inorganic sources. Plant and Soil, Amsterdam, v. 198, n. 2, p. 159-168, 1998.

PRADO, R. M. Nutrição de plantas. São Paulo: Ed. Unesp, 2008.

PROCHNOW, L. I.; ALCARDE, J. C.; CHIEN, S. H. Eficiência agronômica dos fosfatos totalmente acidiculados. In: YAMADA, T.; ABDALLAS, S. R. S. (Eds.). Fósforo na agricultura brasileira. Piracicaba: Potafos, 2004. p. 605-651.

RAIJ, B. V. et al. Análise química para avaliação da fertilidade dos solos tropicais. Campinas: Instituto Agronômico, 2001.

RICHARSON, A. E. et al. Acquisition of phosphorus and nitrogen in the rhizosphere and plant growth promotion by microorganisms. Plant and Soil, Dordrechet, v. 321, n. 1, p. 305-339, 2009.

RODRIGUES, H. J. B. et al. Variabilidade quantitativa de população microbiana associada às condições microclimáticas observadas em solo de floresta tropical úmida. Revista Brasileira de Meteorologia, São Paulo, v. 26, n. 4, p. 629-638, 2011.

SANTOS, D. R.; GATIBONI, L. C.; KAMINSKI, J. Fatores que afetam a disponibilidade do fósforo e o manejo da adubação fosfatada em solos sob sistema plantio direto. Ciência Rural, Santa Maria, v. 38, n. 2, p. 576-586, 2008.

SANTOS, D. H. et al. Produtividade de cana-de-açúcar sob adubação com torta de filtro enriquecida com fosfato solúvel. Pesquisa Agropecuária Tropical, Goiânia, v. 40, n. 4, p. 454-461, 2010.

SILVA, F. A. S.; AZEVEDO, C. A. V. A new version of the Assistat - Statistical Assistance Software. In: WORLD CONGRESS ON COMPUTERS IN AGRICULTURE, 4., 2006, Orlando. Proceedings... Orlando: American Society of Agricultural and Biological Engineers, 2006. p. 393-396.

WOLLUM,A. G. Cultural methods for soil microorganisms. In: PAGE, A. L.; MILLER, R. H.; KEENEY, D. R. (Eds.). Methods of soil analysis. Madison: Soil Science Society of America, 1982. p. 781-802. 